\title{
Dynamics of solitary waves in ultracold gases in terms of observable quantities
}

\author{
L. P. Pitaevskii ${ }^{1,2}$ \\ ${ }^{1}$ Kapitza Institute for Physical Problems RAS, Kosygina 2, 119334 Moscow, Russia \\ ${ }^{2}$ INO-CNR BEC Center and Dipartimento di Fisica, Università di Trento, 38123 Povo, Italy
}

\begin{abstract}
A variety of solitary waves, such as solitons, vortex rings, solitonic vortices, and more complex entities, have recently been predicted to exist. They can move in superfluid ultracold gases along elongated traps. The theoretical description of this motion requires knowledge of the inertial soliton mass and the effective number of particles in it as functions of the soliton energy. While these functions can be calculated by a microscopic theory, it is also possible to express them directly in terms of observable quantities, such as the order parameter phase jump and the particle number depletion in the soliton. In this article, the corresponding equations are derived in a simple and physically clear way and applied to the recently predicted 'magnetic soliton' in mixtures of Bose gases in various spin states.
\end{abstract}

PACS numbers: 03.75.Lm, 3.75.Kk, 67.85.De

DOI: https://doi.org/10.3367/UFNe.2016.08.037891

\section{INTRODUCTION. MACROSCOPIC EQUATIONS OF MOTION OF A SOLITON AND OBSERVABLE QUANTITIES}

The existence of solitary waves - solitons, vortex rings, solitonic vortices, and more complex entities is an important property of quantum gases. The detailed study of the solitary waves has become possible with the development of experimental techniques for confining atomic gases in magnetic and optical traps and cooling them to ultralow temperatures. At such temperatures, a gas becomes super- fluid, and solitary waves can be observed as moving non- decaying objects. (For brevity, in what follows I refer to these waves as solitons.) The essential point is that these excitations, while macroscopic, contain a certain highly perturbed core region, which has a structure dependent on the specific properties of the system and which should be described in microscopic terms. A typical experiment for studying such objects consists in observing their motion in an elongated trap. If the trap is sufficiently long, the problem can be solved in two stages, by first finding the solution for a uniform cylindrical trap and then determining the motion of a soliton in a finite trap applying macroscopic equations of motion. It turns out, however, that soliton dynamics in such a one-dimensional geometry exhibits interesting features due to the occurrence of a jump in the order parameter phase (see Section 2). For example, this jump substantially affects the canonical momentum of the soliton, a fact which was already noted in the classical work of T. Tsuzuki who used the Gross-Pitaevskii (below abbreviated GP) equations to construct a plane ${ }^{1}$ soliton in a dilute Bose-Einstein condensed gas [1].

The soliton equation of motion in an elongated trap

\footnotetext{
${ }^{1}$ Plane in the sense that all the quantities involved are assumed to depend on the $z$ coordinate alone.
}

can be derived from the energy conservation law. A convenient way to do this involves using the soliton velocity $V$ and the chemical potential $\mu$ of the gas as independent variables. The soliton energy should then be determined by employing the thermodynamic potential of the grand canonical ensemble, $E^{\prime}=E_{0}^{\prime}-\mu N$, where $E$ is the energy, and $N$ is the number of particles in the system. For a soliton in a uniform trap, one has $E^{\prime}=E_{0}^{\prime}+\epsilon(V, \mu)$ (where $E_{0}^{\prime}$ is the ground state potential) and $\epsilon(V, \mu)$ is, by definition, the soliton energy. For a weakly nonuniform (i.e. very elongated in the $z$-detection) trap, the energy can be approximated as

$$
\epsilon(V, \mu, z)=\epsilon\left(V, \mu-V_{\text {ext }}(z)\right),
$$

where $z$ denotes the soliton center coordinate, and $V_{\text {ext }}(z)$ is the trap potential. (This is what is known as the local density approximation.) For a harmonic trap, the potential takes the form $V_{\text {ext }}(z)=M \omega_{z}^{2} z^{2} / 2$. The soliton equation of motion is determined by the energy conservation $\epsilon(V, \mu, z)=$ const. 2, 3]. Differentiating Eqn (11) with respect to time and noting that $d z / d t=V$, the equation of motion assumes the form

$$
m_{I} \frac{\partial V}{\partial t}=-N_{S} \frac{\partial V}{\partial z}
$$

where

$$
m_{I}=\frac{1}{V}\left(\frac{\partial \epsilon}{\partial V}\right)_{\mu}, N_{S}=-\left(\frac{\partial \epsilon}{\partial \mu}\right)_{V} .
$$

Here, $m_{I}$ has the meaning of the soliton inertial mass, and $N_{S}$ is the effective number of atoms in the soliton ${ }^{2}$. Thus, knowing the energy (1) allows the determination

\footnotetext{
2 The quantity $m_{I}$ is also often called 'effevtive mass' and denoted $m^{*}, m_{s}$ or $m_{e f f}$.
} 
of the soliton motion in the trap. However, the calculation of $\epsilon$ is a challenging theoretical problem in itself, so it is of great interest whether $m_{I}$ and $N_{S}$ can be expressed directly in terms of observable quantities. It is this question with which this paper is concerned.

I will assume that a superfluid gas is described by a complex order parameter $\Psi$, whose phase $\phi$ defines the superfluid velocity through the equation

$$
\mathbf{v}=\frac{\hbar}{M} \nabla \phi
$$

For a gas of bosons, $\Psi$ is the wave function of the condensate, and $M=m$. For a gas of superfluid fermions, $\Psi$ is the Ginzburg-Landau wave function, and $M=2 m$ because this function is the wave function of superconducting pairs. Here, $m$ is the mass of the atom. Far from the soliton, where the hydrodynamics is valid, the atomic density flux is $\mathbf{j}=\mathbf{n v}$, where $n$ is the gas number density. There are two observable quantities in terms of which I will express $m_{I}$ and $N_{S}$. One is the order parameter phase jump

$$
\Delta \phi=\phi(z=\infty)-\phi(z=-\infty) .
$$

It can be shown [4] that $\phi( \pm \infty)$ is independent of $x$ and $y$. The phase jump $\Delta \phi$ can be (but has not yet been) measured in an interference experiment and arises in a natural way in the numerical calculations of the soliton structure. The other observable quantity is the 'number of depleted atoms in the soliton', namely

$$
N_{D}=\int_{-\infty}^{\infty}\left[n_{1}(z)-n_{1 \infty}\right] d z
$$

where $n_{1}(z)$ is the one-dimensional (i.e., $x-$ and $y$-integrated) atomic number density in the soliton core, and $n_{1 \infty}=n_{1}(z= \pm \infty)$ is the unperturbed density. Usually, the quantity $N_{D}$ is negative, and the soliton is 'dark'. Hence, the term 'depleted'. The quantity $N_{D}$ can be calculated from the observed density distribution in the soliton. Notice that $N_{D} \rightarrow 0$ at $V \rightarrow c$, where $c$ is the speed of sound. Indeed, it is only density perturbations of infinitesimal amplitude that can travel at the speed of sound.

\section{CANONICAL MOMENTUM AND INERTIAL MASS}

The question of the effective mass can be conveniently addressed by first discussing the soliton's momentum. By definition, the momentum is directed along the z-axis and is equal to

$$
P=m \int j_{z} d^{3} x .
$$

It can be verified that the integral in this equation converges for large $\mathrm{z}$ and is therefore uniquely defined. It is a simple matter to express $P$ in terms of $N_{D}$. Consider the soliton to be in the reference frame in which it is at rest, and let the flux of atoms in this frame be $\mathbf{j}^{(0)}$. Because motion in this frame is steady-state, $\mathbf{j}^{(0)}$ is independent of time and the continuity equation takes the form $\nabla \cdot \mathbf{j}^{(0)}=0$. Integrating this equation with respect to $x$ and $y$ gives $\partial_{z} \int j_{z}^{(0)} d x d y=0$. Thus, $\int j_{z}^{(0)} d x d y$ is independent of $\mathrm{z}$ and is equal to its value at $z= \pm \infty$, namely $-n_{1 \infty} V$, because in this frame the liquid outside of the soliton moves with velocity $-V$. On the other hand, the flux in the laboratory frame is, via the Galilean transformation, $j_{z}=j_{z}^{(0)}+V n$. Substituting these formulas into Eqn (7), we find (see Ref. [6])

$$
P=m \int_{-\infty}^{\infty}\left[n_{1}(z)-n_{1 \infty}\right] d z V=m N_{D} V .
$$

I will call the quantity $P$ in (7)-(8) the 'local momentum'. The local momentum becomes clearly zero at $V=0$ and also goes to zero together with $N_{D}$ as the velocity $V$ approaches the speed of sound. The important feature of the one-dimensional geometry considered is that $P$ is not identical to the canonical momentum $P_{C}$ which satisfies the equation

$$
\left(\frac{\partial \epsilon}{\partial P_{C}}\right)_{\mu}=V
$$

or equivalently

$$
m_{I}=\frac{1}{V}\left(\frac{\partial \epsilon}{\partial V}\right)_{\mu}=\left(\frac{\partial P_{C}}{\partial V}\right)_{\mu} .
$$

The physical meaning of the difference between $P$ and $P_{C}$ is currently well understood (see, for example, monograph [5], Ch. 5). Let the soliton move in a trap folded into a toroidal ring of radius $R$. Further, let the radius be so large that the curvature of the trap has no effect on the soliton dynamics. This implies, in fact, the 'thermodynamic limit' for $R \rightarrow \infty$. Upon creating a soliton in a toroidal trap, the wave function should remain unique. The presence of a phase jump Df is in itself a factor that violates this uniqueness. This means that the creation of a soliton occurs with the appearance outside of it of a counterflow which compensates for the phase jump. The momentum of the counterflow makes a contribution to the canonical momentum. The counterflow velocity is calculated from the condition that

$$
\Delta \phi_{c f l}=2 \pi R v_{c f l} M / \hbar=-\Delta \phi,
$$

where account was taken of the fact that outside of the soliton, i.e., for

$$
|z| \gg \xi,
$$

where $\xi$ is the soliton core thickness, the counterflow velocity can be considered constant across the cross section 
of the trap. Accordingly, the counterflow momentum is $P_{c f l}=2 \pi R m n_{1 \infty} v_{c f l}=-\hbar m n_{1 \infty} \Delta \phi / M$. The canonical momentum represents now the total momentum, i.e., equals the sum of $P+P_{c f l}{ }^{3}$ :

$$
P_{C}=m N_{D} V-\hbar n_{1 \infty} \frac{m}{M} \Delta \phi
$$

Differentiating this equation with respect to $V$ and taking into account Eqn (10), we arrive at the sought after relation among $m_{I}, N_{D}$, and $\Delta \phi$ :

$$
m_{I}=m\left(\frac{\partial}{\partial V} N_{D} V\right)_{\mu}-\hbar n_{1 \infty} \frac{m}{M}\left(\frac{\partial}{\partial V} \Delta \phi\right)_{\mu} .
$$

This equation was used in Ref. [6] when studying soliton dynamics in a superfluid Fermi gas under unitarity conditions. The trapped soliton was assumed there to perform only small oscillations, and the difference between $N_{S}$ and $N_{D}$ was not significant (see Section 3).

\section{EFFECTIVE NUMBER OF PARTICLES AND DEPLETION OF PARTICLE NUMBER}

My task now reduces to expressing the effective number of particles $N_{S}$ in a soliton in terms of $N_{D}$ and $\Delta \phi$. For a soliton at rest, the problem is simple: the system is steady-state, so that it is possible to apply the thermodynamic relation for the energy $E^{\prime}$ of the grand canonical ensemble: $N=-\left(\partial E^{\prime} / \partial \mu\right)$, where $N$ is the number of atoms in the system. By definition, the soliton energy is $E^{\prime}(\mu)=E_{0}(\mu)+\epsilon(V=0, \mu)$, where $E_{0}(\mu)$ is the energy in the absence of the soliton, and $N=N_{0}+N_{D}$. Thus, we obtain

$$
N_{D}=-\frac{\partial \epsilon(V=0, \mu)}{\partial \mu}=N_{S} .
$$

This formula, however, does not apply to a moving soliton, because in the laboratory frame the problem is not stationary, and so the question requires a special analysis. Such an analysis has indeed been made in Refs [8, 9] which used $\mu$ and $P_{C}$ as calculation variables. (The distinction between $N_{D}$ and $N_{S}$ was brought to my attention by D M Gangardt.) My hope is that the derivation below will give insight into the physical meaning of the equations obtained. The investigation of this interesting question has long been hindered by the fact, to some extent accidental, that for a plane Tsuzuki soliton the equality $N_{D}=N_{S}$ is also valid at a finite velocity.

\footnotetext{
3 The question of how actually to calculate the momentum is not addressed in this article. For solitary waves comprising quantized vortices, an equivalent equation derived in Ref. [7] is more suitable.
}

In order to apply thermodynamics to a moving soliton, it is necessary to transfer to a reference frame which moves relative the lab frame with velocity $\mathrm{V}$ and where the soliton is at rest. According to the general Galilean transformation rule for mechanical quantities, the soliton energy in this frame is given by

$$
\tilde{\epsilon}(V, \mu)=\epsilon(V, \mu)-P_{C}(V, \mu) V .
$$

Соответственно, изменение числа частиц при рождении солитона, аналогично (15), равно

$$
\Delta N=-\frac{\partial \tilde{\epsilon}(V, \mu)}{\partial \mu}=-\frac{\partial}{\partial \mu}\left(\epsilon-P_{C} V\right)_{V}
$$

This quantity cannot, however, be identified so far with $N_{D}$. Due to the presence of the counterflow, also outside of the soliton the number of particles changes by a finite amount $\Delta N_{c f l}$. As $R \rightarrow \infty$, the density of these particles tends to zero and cannot be measured, so DNcfl should be subtracted from $\Delta N$ :

$$
N_{D}=\Delta N-\Delta N_{c f l} .
$$

The quantity $\Delta N_{c f l}$ is easily calculated using the Bernoulli hydrodynamic equation valid in the superfluid gas outside of the soliton:

$$
\mu_{l}\left(n_{1 \infty}\right)+m \frac{v^{2}}{2}=\mu_{0},
$$

where $\mu_{l}\left(n_{1 \infty}\right)$ is the chemical potential of the gas at rest expressed in terms of its number density, and $v$ is the gas velocity. In the ground state, one has $v=$ $-V$. Upon creating a soliton, $v=-V+v_{c f l}$. Thus, the creation of a soliton changes $\mu_{l}$ by the quantity $\Delta \mu_{l}=m\left[\left(-V+v_{c f l}\right)^{2}-V^{2}\right] / 2 \approx m V v_{c f l}$. The corresponding change in the number density is $\Delta n_{1 \infty}=$ $\left(d \mu / d n_{1 \infty}\right) m V v_{c f l}$, and a change in the number of particles is written out as

$$
\Delta N_{c f l}=\frac{d n_{1 \infty}}{d \mu} m V 2 \pi R v_{c f l}=-\frac{d n_{1 \infty}}{d \mu} V \frac{m}{M} \Delta \phi,
$$

where I have made use of equation (11) for $2 \pi R v_{c f l}$. Substituting Eqns (17) and (20) into Eqn (18), we finally obtain the deficiency in the number of particles in the soliton:

$$
N_{D}=-\frac{\partial}{\partial \mu}\left(\epsilon-P_{C} V\right)_{V}+\frac{d n_{1 \infty}}{d \mu} V \frac{m}{M} \Delta \phi .
$$

Using definition (3) of the effective number of atoms and expression (13) for momentum $P_{C}$, the desired expression of $N_{S}$ in terms of $N_{D}$ and $\Delta \phi$ looks as follows:

$$
N_{S}=N_{D}-m V^{2}\left(\frac{\partial}{\partial \mu} N_{D}\right)_{V}-V \hbar n_{1 \infty} \frac{m}{M}\left(\frac{\partial}{\partial \mu} \Delta \phi\right)_{V} .
$$


For the Tsuzuki soliton, all the considered quantities can be expressed explicitly in analytical form by solving the Gross-Pitaevskii equation. Equations (114), (21) then give

$$
N_{S}=N_{D}=m_{I} / 2
$$

independent of velocity $V$. The same result is obtained by directly integrating (6).

Let us return to (21). We first eliminate $\Delta \phi$ from this equation by making use of Eqn (13) and then proceed by using the following relation between the derivatives, which is valid if canonical equation (9) is satisfied:

$$
\frac{\partial}{\partial \mu}\left(\epsilon-P_{C} V\right)_{V}=\left(\frac{\partial \epsilon}{\partial \mu}\right)_{P_{C}} .
$$

As a result, we arrive at the equation for $N_{D}$ :

$$
\left(1-\frac{m V^{2}}{n_{1 \infty}} \frac{d n_{1 \infty}}{d \mu}\right) N_{D}=-\left[\left(\frac{\partial \epsilon}{\partial \mu}\right)_{P_{C}}+\frac{V}{n_{1 \infty}} \frac{d n_{1 \infty}}{d \mu} P_{C}\right]
$$

or, taking into account the equation for the speed of sound, $c^{2}=\left(n_{1 \infty} / m\right)\left(d \mu / d n_{1 \infty}\right)$, we find in the upshot:

$$
N_{D}=-\frac{(\partial \epsilon / \partial \mu)_{P_{C}}+V P_{C} / m c^{2}}{1-V^{2} / c^{2}} \text {. }
$$

It is in this form that the equation was first presented in Ref. [9], see Eqn (3.16). This equation is convenient to apply when the soliton energy is expressed as a function of $P_{C}$, not of $V$, as is commonly the case with microscopic theories based on exactly solvable models. As for equations, analogous to the mean field theory GP equation, calculations using them involve the variables $\mu, V$. Equation (25) has been employed to calculate $N_{D}$ in a one-dimensional Fermi gas [10]. Notice that by substituting $N_{D}$ from Eqn (26) into Eqn (13) we obtain, by resorting to the canonical momentum definition (9), the equation which defines the phase jump $\Delta \phi$ in terms of the derivatives of $\epsilon\left(P_{C}, \mu\right)$.

\section{NONTRIVIAL EXAMPLE. MAGNETIC SOLITON}

As I have already noted, the application of the obtained relations to a plane soliton in a weakly nonideal Bose gas gives a trivial result (23). There are currently only few analytical solutions that describe solitons. In this section, I will apply the obtained relations to the recently predicted phenomenon of a 'magnetic soliton' in the mixture of two Bose-Einstein condensates residing in different hyperfine triplet states [11]. Such a mixture is described by a system of two coupled Gross-Pitaevskii type equations. However, the problem greatly simplifies if the inequality

$$
\delta \equiv a-a_{12} \ll a
$$

holds, where $a=\sqrt{a_{11} a_{22}}$ and $a_{11} \approx a_{22}$ are scattering lengths for atoms in the same state, and $a_{12}$ is the scattering length for atoms in different states. Under this condition, the equations describing the dynamics of the total gas number density $n=n_{1}+n_{2}$ separate from those for the difference $n_{1}-n_{2}$ of the component number densities. I will consider a symmetric case, in which $a_{11}=a_{22}$ and the unperturbed densities are equal, $n_{1}=n_{2}$. The essential point is that small perturbations of the density $n$ travel at sound speed $c=\sqrt{g n / m}$, where $g$ is the interaction constant equal to $g=4 \pi \hbar^{2} a / m$ in the case of purely one-dimensional motion. The chemical potential is equal to the usual value for a condensate, $\mu=g n$. As for the difference $n_{1}-n_{2}$, which has the meaning of the gas spin polarization, its perturbations travel with the speed of 'spin sound':

$$
c_{s}=\sqrt{\alpha \frac{g n}{m}}=\sqrt{\alpha \frac{\mu}{m}},
$$

where $\alpha=\delta a / 2 a \ll 1$ is the small parameter of the problem, $c_{s} \ll c$. Notice that the total number density $n$ is perturbed weakly. In a similar way, it is possible to construct an analytical solution describing a plane "magnetic soliton', i.e., a localized region in which $n_{1}-n_{2} \neq 0$, and the total number density $n$ is constant in the first approximation. The variables involved are $n_{1}-n_{2}$ и фазы $\phi_{1}$ and $\phi_{2}$ of two order parameters, instead of which it is convenient to introduce the phase difference $\phi_{A}=\phi_{1}-\phi_{2}$ and the phase sum $\phi_{B}=\phi_{1}+\phi_{2}$. In the following, we will only need the energy of the magnetic soliton [11]:

$$
\epsilon_{M}=n \hbar \sqrt{c_{s}^{2}-V^{2}}
$$

and the jump in the phase sum $\phi_{B}$ :

$$
\Delta \phi_{B}=-2 \arccos \left(\frac{V}{c_{s}}\right) \text {. }
$$

Equation (13) for the canonical momentum is readily extended to the case of two condensates:

$$
P_{M C}=m N_{M D} V-\hbar \frac{n}{2} \Delta \phi_{B} .
$$

At this point, we should take into account that the total number density $n$ also remains constant within the soliton, which means that the first term in this equation is small and can be disregarded. (It can be shown that $\left.\left|N_{M D}\right| V \sim \alpha \hbar n \Delta \phi_{B}.\right)$ Thus, we obtain

$$
P_{M C} \approx-\hbar \frac{n}{2} \Delta \phi_{B}=-\hbar n \arccos \left(\frac{V}{c_{s}}\right) .
$$

The canonical momentum of the magnetic soliton is almost completely determined by the counterflow. From Eqn (32), the following simple expression for the soliton energy as a function of the momentum can be derived:

$$
\epsilon_{M}=n \hbar c_{s}\left|\sin \left(\frac{P_{M C}}{\hbar n}\right)\right|,-\frac{\pi \hbar n}{2} \leqslant P_{M C} \leqslant \frac{\pi \hbar n}{2} .
$$


Differentiating expressions (29) yields the soliton inertial mass $m_{M I}$ and the effective number of particles $N_{M S}$ :

$$
\begin{gathered}
m_{M I}=-\frac{n \hbar}{\sqrt{c_{s}^{2}-V^{2}}}, \\
N_{M S}=-\frac{\hbar}{g}\left[\sqrt{c_{s}^{2}-V^{2}}+\frac{c_{s}^{2}}{2 \sqrt{c_{s}^{2}-V^{2}}}\right] .
\end{gathered}
$$

While both these quantities diverge on approaching the magnetic sound speed, their ratio (which enters the equation of motion (2) ) remains finite:

$$
\frac{N_{M S}}{m_{M I}}=\frac{1}{\mu}\left[\frac{3}{2} c_{s}^{2}-V^{2}\right] .
$$

Let us now evaluate the depletion of the number of particles $N_{M D}$ in a magnetic soliton. Equation (21) for the case of two condensates takes the form

$$
N_{M D}=-\frac{\partial}{\partial \mu}\left(\epsilon-P_{C} V\right)_{V}+\frac{d n_{1 \infty}}{d \mu} V \frac{m}{2} \Delta \phi
$$

or, expressing $P_{C}$ in terms of $\Delta \phi$ according to Eqn (32), we obtain

$$
N_{M D}=-\frac{\partial \epsilon}{\partial \mu}-n V \frac{m}{2} \frac{\partial}{d \mu} \Delta \phi .
$$

A simple calculation gives

$$
N_{M D}=-\frac{3 \hbar}{2 g} \sqrt{c_{s}^{2}-V^{2}} .
$$

We see that, unlike the Tsuzuki soliton, $N_{M S}$ and $N_{M D}$ behave totally differently as $V \rightarrow c_{s}$. At $V=0$, these quantities are equal (as they should be).

\section{Derivation the relation between $N_{S}$ and $N_{D}$ directly from equations}

In this section, we will derive equation (21) directly from an equation that generalizes the Gross-Pitaevskii equation. Such generalized equations are widely applied in soliton theory, if the system can be described by a mean field theory. Among such problems is, in particular, that of applying the GP equation to the study of solitary waves in a cylindrical trap. The point is that simple relation (23) is valid only for a plane Tsuzuki soliton. If, however, a radial confining potential is present, then even in the case of an ordinary soliton the equations obtained above should be used, let alone more complex solitary waves that exist in such a geometry [4, 12].

I will assume that the system is described by the energy functional $H\left[\Psi, \Psi^{*}\right]$, where $\Psi$ is the order parameter (or the wave function) $\Psi(t, \mathbf{r})=\sqrt{n} e^{i \phi}$, so that $\mathbf{j}=n \frac{\hbar}{m} \nabla \phi$. (I set $M=m$, thus limiting the problem to the case of bosons.)
The equation for $\Psi$ is obtained by varying of the the energy functional of the grand canonical distribution $H^{\prime}\left[\Psi, \Psi^{*}\right]$ over $\Psi^{*}$

$$
i \hbar \partial_{t} \Psi=\frac{\delta H^{\prime}}{\delta \Psi^{*}}
$$

where $H^{\prime}\left[\Psi, \Psi^{*}\right]$ is

$$
H^{\prime}\left[\Psi, \Psi^{*}, \mu\right]=H\left[\Psi, \Psi^{*}\right]-E_{0}^{\prime}-\mu \int|\Psi|^{2} d^{3} x
$$

For a running soliton, the solution is $\Psi=\Psi_{S}(z-V t)$, so that the equation can be rewritten as

$$
-i \hbar V \partial_{z} \Psi_{S}=\frac{\delta H}{\delta \Psi^{*}}
$$

Let us now determine $N_{S}$ and $N_{D}$ in terms $H^{\prime}$. The energy of the gas in the presence of a soliton is $\varepsilon=$ $H^{\prime}\left[\Psi_{S}, \Psi_{S}^{*}, \mu\right]$ and $N_{S}=-\frac{d}{d \mu} H^{\prime}\left[\Psi_{S}, \Psi_{S}^{*}, \mu\right]$. On the other hand, differentiating Eqn (40) at constant obviously gives

$$
\frac{d}{d \mu}\left(H^{\prime}\right)_{\Psi}=-\int\left(|\Psi|^{2}-n_{\infty}\right) d^{3} x,
$$

because $d E_{0}^{\prime} / d \mu=-N$. Thus, we obtain

$$
N_{D}=-\frac{d}{d \mu}\left(H^{\prime}\right)_{\Psi=\Psi_{S}}
$$

Differentiation of the functional $H^{\prime}\left[\Psi_{S}, \Psi_{S}^{*}, \mu\right]$ yields

$$
N_{S}=N_{D}-\int\left(\frac{\delta H^{\prime}}{\delta \Psi^{*}} \frac{d \Psi^{*}}{d \mu}+c . c .\right) d^{3} x
$$

where the replacement $\Psi \rightarrow \Psi_{S}$ should be made after differentiation. Eliminating the variational derivatives with the help (41) results in

$$
N_{S}=N_{D}-2 \hbar V \operatorname{Im} \int\left(\partial_{\mathrm{z}} \Psi_{\mathrm{S}} \frac{\mathrm{d} \Psi_{\mathrm{S}}^{*}}{\mathrm{~d} \mu}\right) \mathrm{d}^{3} \mathrm{x} .
$$

Notice that, given this equation, no additional calculations are needed to show that for a plane Tsuzuki soliton $N_{S}=N_{D}$. As shown in Ref. [5], Eqn (5.56)] the solution in this case can be written out in a form in which $\operatorname{Im} \Psi_{\mathrm{S}}$ is independent of $z$ and $\mu$, bringing the second term in Eqn (43) to zero. Substituting $\Psi_{S}=\sqrt{n} e^{i \phi}$ into Eqn (43) gives, after simple algebra, the following expression

$$
N_{S}-N_{D}=-\hbar V \int\left[\frac{d}{d \mu}\left(n \partial_{z} \phi\right)-\partial_{z}\left(n \frac{d \phi}{d \mu}\right)\right] d^{3} x
$$

In the second term of this equation, we can integrate with respect to $z$ to obtain

$$
\int \partial_{z}\left(n \frac{d \phi}{d \mu}\right) d^{3} x=n_{1 \infty} \frac{d \Delta \phi}{d \mu}
$$


where account was taken of the fact that $\phi(z \rightarrow \pm \infty)$ is independent of $x$ and $y$. Using the local momentum definition (7), we arrive at the equation

$$
N_{S}-N_{D}=-V \frac{d P}{d \mu}+n_{1 \infty} V \hbar \frac{d \Delta \phi}{d \mu}
$$

which taking into account Eqn (13), is identical to Eqn (21).

\section{CONCLUSION}

The theoretical description of solitary waves is complex and requires a microscopic theory, because the characteristic core thickness is on the order of the correlation length of particles in the system. Accordingly, the comparison of theory with experiment provides an important insight into the validity of the theoretical approach used. The study of soliton motion in an elongated trap is the most important of the experiments available. Comparing theory with experiment using the observables $N_{D}$ and $\Delta \phi$ opens new possibilities. This is especially true of numerical simulations, when these two quantities are easily determined and comparison with the obtained equation of motion makes it possible to test the self-consistency of calculations and their underlying theory, as well as allowing the estimation of the computation accuracy.

\section{AFTERWORD}

The present paper has been submitted to the special October 2016 issue of Uspekhi Fizicheskikh Nauk [PhysicsUspekhi] journal on the occasion of the 100th anniversary of the birth of V. L. Ginzburg. He was a remarkable physicist recognized for his classical contributions to diverse areas of theoretical physics. My personal communications with him contributed much to my development as a scientist, and I was always an admirer of his personal qualities. The beginning of our friendship - one that lasted uninterrupted until his death - was our joint work on the theory of superfluidity in ${ }^{4} \mathrm{He}$ near the $\lambda$ point [13, 14]. From the very beginning I was greatly impressed by the clarity of his thought and by the total absence of pomposity in his manners. A second intersection of our activities had to do with the theory of van der Waals forces. The theory of these forces, which I E Dzyaloshinskii, E M Lifshitz, and I [15, 16] developed for an electromagnetic field in energy-absorbing dielectrics, was formulated in terms of quantum field theoretical diagrammatic techniques. Ginzburg asked me then whether this could be done without applying this technique, and I was firmly confident it could not. Shortly thereafter, however, he devised a clever way to overcome absorptionrelated difficulties and developed (with Yu S Barash) a complete theory [17] which, among other things, made it possible to get rid of some cumbersome calculations.
The theory of solitons, which is the subject of this article, was to my knowledge never among his interests. But the theory of solitons in superfluid Fermi systems always and inevitably uses the boson order parameter, i.e., the Ginzburg - Landau wave function. It should be noted, by the way, that the Ginzburg - Landau theory only increases in importance as time goes on. Of course and alas, nothing can replace direct personal communication P but a soothing fact is that V L Ginzburg's work remains and will be of service to future generations of physicists. Of special note is V L Ginzburg's role as the Chief Editor of the Uspekhi Fizicheskikh Nauk [Physics - Uspekhi] journal [18]. His continual work in the journal lasted, literally, to the last day of his life, and it was $\mathrm{V}$ L Ginzburg's constant effort to pursue impartiality and firm principles in the editorial policy.

\section{Acknowledgments}

I am grateful to D M Gangardt, F Dalfovo, and S Giorgini for fruitful discussions of the subject. This work was supported by ERC through a QGBE grant, by a QUIC grant from the Horizon2020 FET program, and by Provincia Autonoma di Trento.

[1] Tsuzuki T J. Low Temp. Phys. 4, 441 (1971)

[2] Busch Th and Anglin J R Phys. Rev. Lett. 84, 2298 (2000)

[3] Konotop V V and Pitaevskii L P Phys. Rev. Lett. 93, 240403 (2004)

[4] Komineas, S and Papanicolaou N Phys. Rev. A 68043617 (2003)

[5] Pitaevskii L P and Stringari S, Bose-Einstein Condensation and Superfluidity, (Orford University Press, New York, 2016).

[6] Scott R G, Dalfovo F, Pitaevskii L P, and Stringari S Phys. Rev. Lett. 106, 185301 (2011)

[7] Pitaevskii JETP 1191097 (2014); arXiv:1311.4693

[8] Schecter M, Gangardt D M, and Kamenev A Ann. Phys. (N. Y) 327639 (2012)

[9] Campbell A S "Mobile impurities in one dimentional quantum liquids." PhD thesis University of Birmingham (2013)

[10] Shamailov S B and Brand J. new J. Phys 18, 075004 (2016); arXiv:1603.04864

[11] Qu Chunlei, Pitaevskii L P, and Stringari S Phys. Rev. Lett. 116, 160402 (2016)

[12] Brand R and Reinhardt W P Phys. Rev. A 65, 043612 (2002)

[13] Ginzburg V L, Pitaevskii L P Sov. Phys. JETP 7858 (1958)

[14] Ginzburg V L Phys. Usp. 471155 (2004)

[15] Dzyaloshinskii I E, Pitaevskii L P Sov. Phys. JETP 9 1282 (1959)

[16] Dzyaloshinskii I E, Lifshitz E M, Pitaevskii L P Sov. Phys. Usp. 4153 (1961) 
[17] Barash Yu S, Ginzburg V L Sov. Phys. Usp. 27467 (1984)

[18] Ginzburg V L Phys. Usp. 52530 (2009) 